\title{
Amplitude Scale Estimation for Quantization-Based Watermarking
}

\author{
Ivo D. Shterev and Reginald L. Lagendijk, Senior Member, IEEE
}

\begin{abstract}
In this paper we propose a maximum likelihood technique to combat amplitude scaling attacks within a quantizationbased watermarking context. We concentrate on operations that are common in many applications and at the same time devastating to this class of watermarking schemes, namely, amplitude scaling in combination with additive noise. First we derive the probability density function of the watermarked and attacked data in the absence of subtractive dither. Next we extend these models to incorporate subtractive dither in the encoder. The dither sequence is primarily used for security purposes, and the dither is assumed to be known also to the decoder. We design the dither signal statistics such that an attacker having no knowledge of the dither cannot decode the watermark. Using an approximation of the probability density function in the presence of subtractive dither, we derive a maximum likelihood procedure for estimating amplitude scaling factors. Experiments are performed with synthetic and real audio signals, showing the feasibility of the proposed approach under realistic conditions.
\end{abstract}

Index Terms-Maximum likelihood estimation, probability of error, quantization, statistics, subtractive dither, watermarking.

\section{INTRODUCTION}

W ATERMARKING is the process of imperceptibly embedding a message (watermark) into a host signal (audio, video). The resulting signal is called a watermarked signal. The message should introduce only tolerable distortion to the host signal and it should be recoverable by the intended receiver after signal processing operations on the watermarked data.

Watermarking schemes based on quantization theory have recently emerged as a result of information theoretic analysis [1]-[4]. In terms of additive noise attacks, these schemes have proven to perform better than traditional spread-spectrum watermarking because they can completely cancel the host signal interference, which makes them invariant to the host signal. The existence of good lattices in high dimensions [5] that can be directly and efficiently implemented has made quantization-based schemes of practical interest.

Lattice-based schemes are vulnerable to amplitude scale attacks because these attacks introduce mismatch between the encoder and the decoder lattice volumes. Furthermore, amplitude scaling induces a large amount of distortion with respect to the mean squared error but does not cause significant perceptual

Manuscript received May 30, 2005; accepted January 10, 2006. This work was supported by the Technology Foundation STW, Applied Science Division of NWO, and Technology Program of the Ministry of Economic Affairs. The associate editor coordinating the review of this manuscript and approving it for publication was Prof. Anuj Srivastava.

The authors are with the Information and Communication Theory Group, Faculty of Electrical Engineering, Mathematics, and Computer Science, Delft University of Technology, 2628 CD Delft, The Netherlands (e-mail: i.shterev@gmail.com; r.1.lagendijk@ewi.tudelft.nl).

Digital Object Identifier 10.1109/TSP.2006.881216 degradations. Such operation on watermarked signals is quite common in many applications. One example is audio play-out and capturing, where the watermarked signal is passed through a digital-to-analog converter, transmitted through an analog noisy channel, captured by a microphone, and converted back to a digital representation. Clearly the microphone will capture a less powerful and degraded watermarked signal, which has led us to model the noisy channel as an amplitude scaling operation followed by additive noise. In this paper, we concentrate on operations consisting of amplitude scaling followed by additive white Gaussian noise (AWGN), often called scale AWGN channel.

Several techniques are known in the literature for combating amplitude scale attacks. One of the approaches is based on designing watermarking codes that are invariant to amplitude scale operations, such as modified trellis codes [6], order-preserving lattice codes [7], and rational dither modulation [8]. Another approach is based on estimating the nonadditive operations and inverting them prior to watermark decoding, using pilot signals [9] or blind estimation [10]-[12]. More recently, an iterative estimation procedure in combination with error-correcting codes was proposed [13], which proved to perform well even for low watermark-to-noise ratios (WNRs). The advantage of the approach in [9] is the ability to estimate the scaling factor from a small number of signal samples, which makes the estimation procedure applicable in situations where the scaling factor slowly varies. The disadvantage of the method is that the pilot signals consume part of the capacity of the watermarking system. The method proposed in [11] performs well for low WNR but lacks security, in the sense that an attacker knowing the distortion of the embedder is able to estimate the scaling factors and decode the watermark. The methods based on invariant codes give small probability of error with respect to amplitude scale attacks at the expense of increased probability of error [8], [7] with respect to additive noise attacks and reduced payload [6].

In this paper, we propose a maximum likelihood (ML) approach for estimating amplitude scaling factors. Our estimation technique is blind and only assumes knowledge of the watermark message priors. No knowledge of the position of the message bits in the watermark bitstream is required. We also introduce subtractive dither [14] in the encoder. The realization of the dither is assumed to be known to the decoder. An application of subtractive dither to watermarking appeared first in [15], but with no theoretical analysis of the system security. In this paper, we design the dither statistics such that an attacker without knowing the dither realization is not able to decode the watermark. Thus the dither serves as the key ensuring security of the system.

This paper is organized as follows. In Section II, we formulate the attack channel, watermark encoder, and decoder. In Section III, we derive the probability density function (pdf) of 


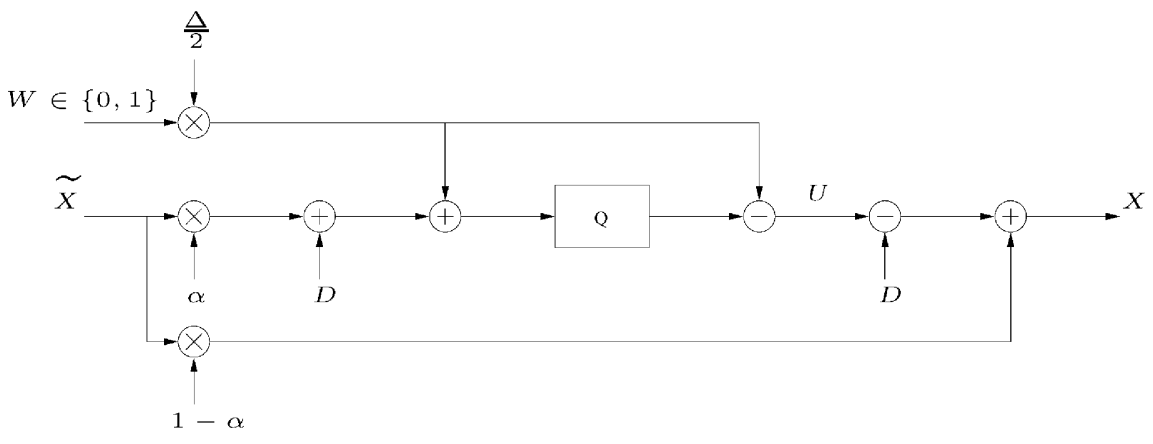

Fig. 1. Watermark encoder.

the received data in the absence of dither [11], as a preliminary step. Then, we extend these pdf models to incorporate subtractive dither. In Section IV, we give approximations to the pdf models for the case when the dither variance is much smaller than the host signal variance. In Section V, we give conditions for the dither sequence statistics such that a given level of security is achieved and at the same time the dither variance is as small as possible, using the probability of error of the watermarking system as an objective function. A description of the ML estimation procedure is given in Section VI. Section VII contains experimental results with synthetic and real audio host signals, and Section VIII concludes this paper.

\section{MATHEMATICAL FORMULATION}

In this paper, we focus on the most popular quantization-based watermarking scheme: scalar quantization index modulation (QIM). Throughout this paper, random variables are denoted by capital letters and their realizations by the respective small letters. The notation $X \sim f_{X}(x)$ indicates that the random variable $X$ has a pdf $f_{X}(x)$.

Fig. 1 shows the watermark encoder, where $W \in\{0,1\}$ denotes the message bits that are embedded in the host data, $\widetilde{X}$ is the host signal itself with a variance $\sigma_{\widetilde{X}}^{2}, X$ is the watermarked signal, and $D \sim f_{D}(d)$ is the dither sequence with a variance $\sigma_{D}^{2}$. The statistics of the dither sequence will be derived in Section V. The variable $U$ is the output of the quantizer. $Q(\cdot)$ denotes uniform quantization with step size $\Delta$. The quantization noise, which is the difference between the quantizer input and output, is defined as

$$
\begin{aligned}
N_{1} & =\alpha \widetilde{X}+D-Q(\alpha \tilde{X}+D) \\
& =\alpha \widetilde{X}-(X-(1-\alpha) \widetilde{X}) \\
& =\widetilde{X}-X
\end{aligned}
$$

where $\alpha$ is a coefficient to be defined later.

From (1), we see that the watermark $\widetilde{X}-X$ and the quantization noise are equal. The quantizer input-output characteristic is shown in Fig. 2 for the watermark message $W \in\{0,1\}$. The output of the quantizer can be written as

$$
U= \begin{cases}k \Delta, & \text { if } W=0 \\ (2 k+1) \frac{\Delta}{2}, & \text { if } W=1\end{cases}
$$

where $k \in(-\infty, \infty)$ is an integer.

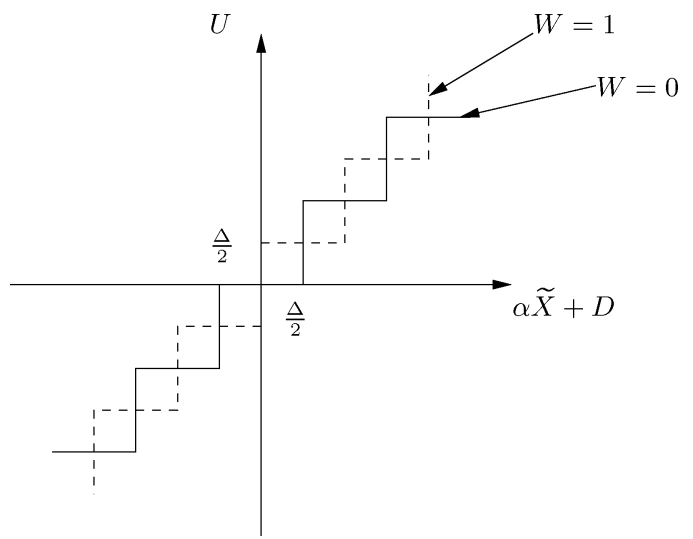

Fig. 2. Quantizer input-output characteristics.

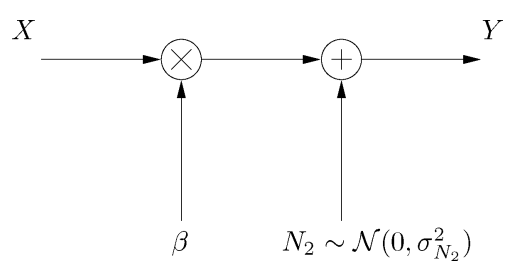

Fig. 3. Attack channel.

The attack channel is shown in Fig. 3. It consists of the constant amplitude scale factor $\beta$ and the noise $N_{2} \sim \mathcal{N}\left(0, \sigma_{N_{2}}^{2}\right)$. The noise $N_{2}$ is independent of $\widetilde{X}$ and $N_{1}$. We choose the coefficient $\alpha=\sigma_{N_{1}}^{2} /\left(\sigma_{N_{1}}^{2}+\sigma_{N_{2}}^{2}\right)$ as in [4], where $\sigma_{N_{1}}^{2}$ is the variance of $N_{1}$. Other choices for $\alpha$ are also possible [16].

The attacked (received) signal $Y$ can be written in the following way:

$$
\begin{aligned}
Y & =\beta X+N_{2} \\
& =\beta(U-D+(1-\alpha) \widetilde{X})+N_{2} .
\end{aligned}
$$

Using the relation $\alpha \tilde{X}=U-D+N_{1}$, we obtain the received data $Y$ in terms of $N_{1}, N_{2}$, and the watermark-bearing signal $U$

$$
Y=\frac{\beta}{\alpha}\left(U-D+(1-\alpha) N_{1}\right)+N_{2} .
$$

The watermark decoder is shown in Fig. 4. From the received signal $Y$, the decoder first performs maximum a posteriori probability estimation of the signal $U-D$, which under mild as- 


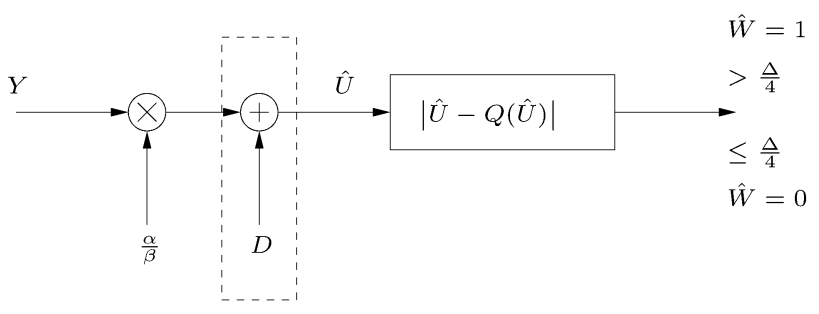

Fig. 4. Watermark decoder.

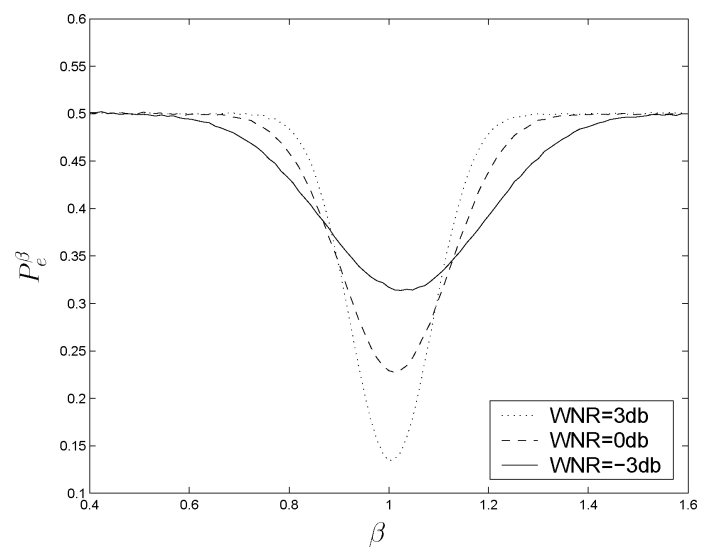

Fig. 5. The effect of $\beta$ on probability of error $P_{e}^{\beta}$ for $D=0$. Experiments are performed for $\widetilde{X} \sim \mathcal{N}(0,1), N_{2} \sim \mathcal{N}\left(0, \sigma_{N_{2}}^{2}\right)$, and DWR $=20 \mathrm{~dB}$.

sumptions [1] is equivalent to multiplication by $\alpha / \beta .^{1}$ Then the decoder adds the dither $D$, obtaining

$$
\begin{aligned}
\hat{U} & =\frac{\alpha}{\beta} Y+D \\
& =U+(1-\alpha) N_{1}+\frac{\alpha}{\beta} N_{2} .
\end{aligned}
$$

The decoder then computes the absolute value of the quantization noise $|\hat{U}-Q(\hat{U})|$ and makes an estimate of the embedded watermark in the following way:

$$
\hat{W}= \begin{cases}0, & \text { if }|\hat{U}-Q(\hat{U})| \leq \frac{\Delta}{4} \\ 1, & \text { if }|\hat{U}-Q(\hat{U})|>\frac{\Delta}{4}\end{cases}
$$

Throughout this paper, we denote $W N R=$ $10 \log \left(\sigma_{N_{1}}^{2} / \sigma_{N_{2}}^{2}\right)$, and the document-to-watermark ratio DWR $=10 \log \left(\sigma_{\widetilde{X}}^{2} / \sigma_{N_{1}}^{2}\right)$.

Experimental results of the effect of unknown $\beta$ on probability of error are shown in Fig. 5. We can see that the amplitude scale attack is more devastating at high WNR. At low WNR, the effect of the attack is less pronounced because the probability of error is already quite large for $\beta=1$.

\section{PDF MODELS}

In this section, we first derive exact pdf models of the watermarked and attacked signals in the absence of dither $(D=0)$.

${ }^{1}$ Here we assume that we are able to perfectly estimate $\beta$
The derivations are used as a base for deriving pdf models in the presence of dither $(D \neq 0)$, on which the ML estimation relies.

A. Case $D=0$

From Fig. 1, the pdf of the watermarked data $X$ is given as

$$
f_{X}(x)=f_{X \mid W=0}(x) \operatorname{Pr}[W=0]+f_{X \mid W=1}(x) \operatorname{Pr}[W=1]
$$

where $\operatorname{Pr}[W=0]$ and $\operatorname{Pr}[W=1]$ are the probabilities of occurrence of bit 0 and 1 , respectively, and $f_{X \mid W=0}(x)$ and $f_{X \mid W=1}(x)$ are the conditional pdfs of the watermarked data corresponding to $W=0$ and $W=1$, respectively.

Taking $\beta$ and $N_{2}$ into account and using the fact that for any $\beta>0$, we have $f_{\beta X \mid W}(x)=(1 / \beta) f_{X \mid W}(x / \beta)$, we obtain the pdf of the received data $Y$ as

$$
\begin{aligned}
f_{Y}(y)= & f_{N_{2}}\left(n_{2}\right) * f_{\beta X \mid W=0}(x) \operatorname{Pr}[W=0] \\
& +f_{N_{2}}\left(n_{2}\right) * f_{\beta X \mid W=1}(x) \operatorname{Pr}[W=1] \\
= & f_{N_{2}}\left(n_{2}\right) *\left[\frac{1}{\beta} f_{X \mid W=0}\left(\frac{x}{\beta}\right) \operatorname{Pr}[W=0]\right] \\
& +f_{N_{2}}\left(n_{2}\right) *\left[\frac{1}{\beta} f_{X \mid W=1}\left(\frac{x}{\beta}\right) \operatorname{Pr}[W=1]\right]
\end{aligned}
$$

where the convolution $*$ follows from the independence between $\beta X$ and $N_{2}$.

We derive the expression for $f_{X \mid W=0}(x)$. The derivation of $f_{X \mid W=1}(x)$ follows using similar reasoning.

Let us consider the case where the input to the quantizer is in the $k$ th quantization cell, i.e., the output of the quantizer is $U=k \Delta$. We have

$$
\Delta\left(k-\frac{1}{2}\right)<\alpha \widetilde{X}<\Delta\left(k+\frac{1}{2}\right) .
$$

Multiplying all sides by the positive term $(1-\alpha) / \alpha$, we get

$$
\frac{(1-\alpha) \Delta}{\alpha}\left(k-\frac{1}{2}\right)<(1-\alpha) \tilde{X}<\frac{(1-\alpha) \Delta}{\alpha}\left(k+\frac{1}{2}\right) .
$$

Adding $k \Delta$ to all sides and reorganizing, we obtain

$$
\frac{\Delta}{\alpha}\left(k-\frac{1-\alpha}{2}\right)<(1-\alpha) \tilde{X}+k \Delta<\frac{\Delta}{\alpha}\left(k+\frac{1-\alpha}{2}\right) .
$$

We define the indicator function

$$
I_{A_{k \mid W=0}}(x)= \begin{cases}1, & \text { if } x \in A_{k \mid W=0} \\ 0, & \text { if } x \notin A_{k \mid W=0}\end{cases}
$$

where

$$
A_{k \mid W=0}=\left[\frac{\Delta}{\alpha}\left(k-\frac{1-\alpha}{2}\right), \frac{\Delta}{\alpha}\left(k+\frac{1-\alpha}{2}\right)\right] .
$$

Therefore, the pdf of $(1-\alpha) \widetilde{X}+k \Delta$ over the support set $A_{k \mid W=0} \quad$ is $\quad 1 /(1-\alpha) f_{\widetilde{X}}((x-k \Delta) /(1-\alpha)) I_{A_{k \mid W=0}}(x)$. Recognizing that $(1-\alpha) \widetilde{X}+k \Delta$ is the watermarked data $X$ for a particular $k$, we can find the pdf of $X$ by summing over $k$. Thus we have

$$
f_{X \mid W=0}(x)=\sum_{k=-\infty}^{\infty} \frac{1}{1-\alpha} f_{\widetilde{X}}\left(\frac{x-k \Delta}{1-\alpha}\right) I_{A_{k \mid W=0}}(x) .
$$




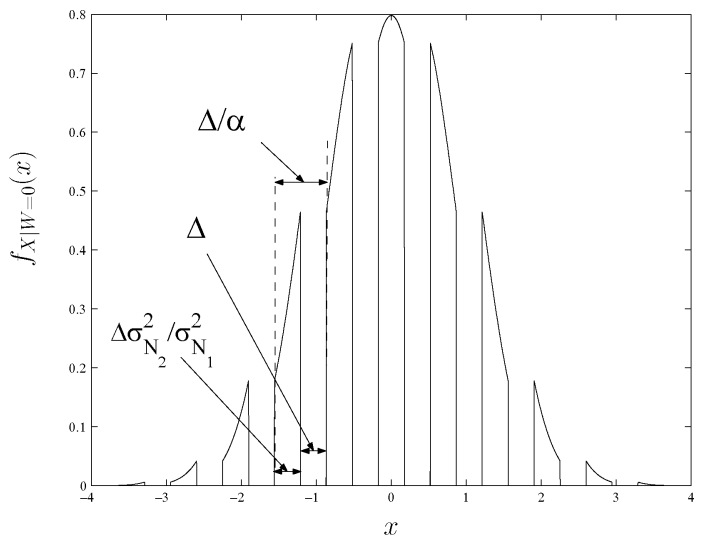

Fig. 6. Graph of $f_{X \mid W=0}(x)$. Chosen settings are $\widetilde{X} \sim \mathcal{N}(0,1), N_{2} \sim$ $\mathcal{N}(0,0.01), \sigma_{N_{1}}^{2}=0.01$, and $\Delta \approx \sqrt{0.12}$.

In the same fashion we can express the pdf of the watermarked data for $W=1$ as

$$
f_{X \mid W=1}(x)=\sum_{k=-\infty}^{\infty} \frac{1}{1-\alpha} f_{\widetilde{X}}\left(\frac{x-\frac{2 k+1}{2} \Delta}{1-\alpha}\right) I_{A_{k \mid W=1}}(x)
$$

where

$$
A_{k \mid W=1}=\left[\frac{\Delta}{\alpha}\left(k+\frac{\alpha}{2}\right), \frac{\Delta}{\alpha}\left(k+\frac{2-\alpha}{2}\right)\right] .
$$

An illustration of (14) is shown in Fig. 6.

Referring to the above equations, we can now take the scaling factor $\beta$ into account

$$
\begin{aligned}
f_{\beta X \mid W=0}(x)= & \frac{1}{\beta(1-\alpha)} \sum_{k=-\infty}^{\infty} f_{\widetilde{X}}\left(\frac{x-k \beta \Delta}{\beta(1-\alpha)}\right) \\
& \times I_{A_{k \mid \beta, W=0}}(x) \\
f_{\beta X \mid W=1}(x)= & \frac{1}{\beta(1-\alpha)} \sum_{k=-\infty}^{\infty} f_{\widetilde{X}}\left(\frac{x-\frac{2 k+1}{2} \beta \Delta}{\beta(1-\alpha)}\right) \\
& \times I_{A_{k \mid \beta, W=1}}(x)
\end{aligned}
$$

where the indicator sets are given as

$$
\begin{aligned}
& A_{k \mid \beta, W=0}=\left[\frac{\beta \Delta}{\alpha}\left(k-\frac{1-\alpha}{2}\right), \frac{\beta \Delta}{\alpha}\left(k+\frac{1-\alpha}{2}\right)\right] \\
& A_{k \mid \beta, W=1}=\left[\frac{\beta \Delta}{\alpha}\left(k+\frac{\alpha}{2}\right), \frac{\beta \Delta}{\alpha}\left(k+\frac{2-\alpha}{2}\right)\right] .
\end{aligned}
$$

An illustration of (17) is shown in Fig. 7. The regular pattern that carries information about the quantity $\beta \Delta / \alpha$ in the pdf of the watermarked data can clearly be seen. Reference [9] exploits similar modeling.

Finally, an illustration of (8) with $\beta=1$ and $\operatorname{Pr}[W=0]=1$ is given in Fig. 8.

\section{B. Case $D \neq 0$}

In this section, we assume that the dither is present in the watermarking system as shown in Figs. 1 and 4. Since in the presence of subtractive dither $f_{X}(x)$ will be perturbed by $D$, it

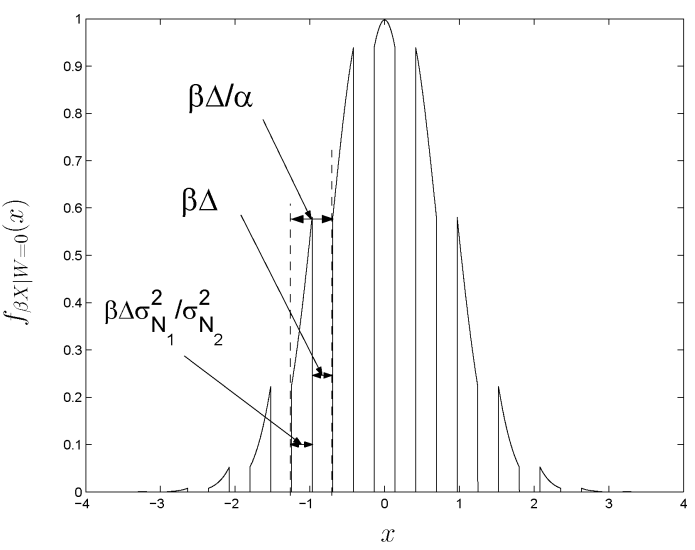

Fig. 7. Graph of $f_{\beta X \mid W=0}(x)$. Chosen settings are $\widetilde{X} \sim \mathcal{N}(0,1), N_{2} \sim$ $\mathcal{N}(0,0.01), \sigma_{N_{1}}^{2}=0.01, \Delta \approx \sqrt{0.12}$, and $\beta=0.8$.

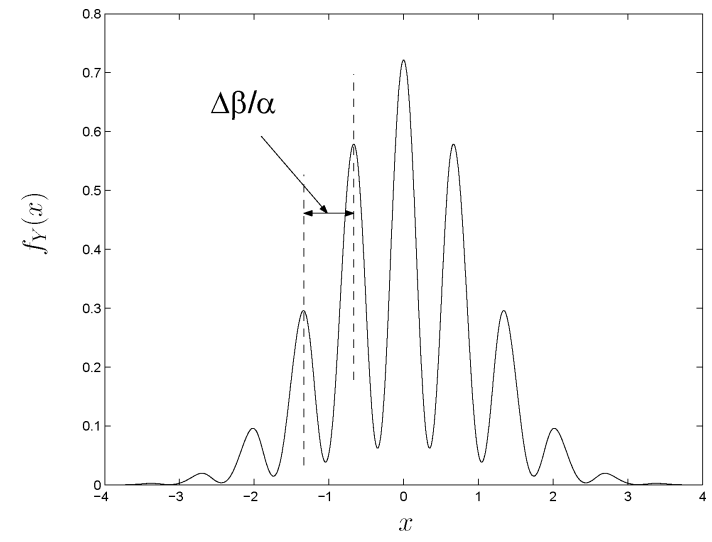

Fig. 8. Graph of $f_{Y}(x)$ with $\operatorname{Pr}(W=0)=1$. Chosen settings are $\widetilde{X} \sim$ $\mathcal{N}(0,1), N_{2} \sim \mathcal{N}(0,0.01), \sigma_{N_{1}}^{2}=0.01$, and $\beta=1$.

is difficult to derive a useful exact mathematical expression for it. That is why we choose to manipulate $X$ in a convenient way, having knowledge of $D$, so that we are able to mathematically describe the structure of the pdf of the resulting random variable.

For simplicity, we will assume that only message $W=0$ is embedded, therefore working only with the first part of (8). Extension to the more general case of embedding zeros and ones is straightforward: use the whole expression (8). Using the same reasoning and notation as in the previous subsection, we derive the pdf models in the presence of subtractive dither.

Referring to Fig. 1, let us assume that $\operatorname{Pr}[W=0]=1$ and $\alpha \widetilde{X}+D$ belongs to the $k$ th quantization cell, i.e.,

$$
\Delta\left(k-\frac{1}{2}\right)<\alpha \widetilde{X}+D<\Delta\left(k+\frac{1}{2}\right) .
$$

Multiplying by $(1-\alpha) / \alpha$ and adding $k \Delta$, we obtain

$$
\begin{aligned}
\frac{\Delta}{\alpha}\left(k-\frac{1-\alpha}{2}\right) & <(1-\alpha) \widetilde{X}+k \Delta+\frac{1-\alpha}{\alpha} D \\
& <\frac{\Delta}{\alpha}\left(k+\frac{1-\alpha}{2}\right) .
\end{aligned}
$$

Recognizing that the leftmost and rightmost parts of (22) are the indicator set $A_{k \mid W=0}$ as given by (13) and taking into account the fact that $(1-\alpha) \widetilde{X}+k \Delta+((1-\alpha) / \alpha) D=X+(1 / \alpha) D$ 
(see Fig. 1), we can write the pdf of $X+(1 / \alpha) D$ for a particular $k$ as

$$
f_{X+\frac{1}{\alpha} D}^{\prime}(x)=f_{(1-\alpha) \widetilde{X}+k \Delta+\frac{1-\alpha}{\alpha} D}(x) I_{A_{k} \mid W=0}(x) .
$$

Generalizing for all $k$, we have

$$
f_{X+\frac{1}{\alpha} D}(x)=\sum_{k=-\infty}^{+\infty} f_{(1-\alpha) \widetilde{X}+k \Delta+\frac{1-\alpha}{\alpha} D}(x) I_{A_{k} \mid W=0}(x) .
$$

Equation (24) is the key expression for the estimation procedure in the presence of subtractive dither. We can see that although $X$ is perturbed by the dither, if we add the term $(1 / \alpha) D$ to the watermarked signal, we are able to obtain a signal that has a pdf with an indicator function equal to that when no dither is used. In other words, we are able to recover the structure of the watermarked signal pdf by the use of the dither.

Taking into account $\beta$ and the additive noise $N_{2}$, we now have

$$
f_{Y+\frac{\beta}{\alpha} D}\left(y+\frac{\beta}{\alpha} d\right)=f_{N_{2}}\left(n_{2}\right) * f_{\beta X+\frac{\beta}{\alpha} D}\left(x+\frac{\beta}{\alpha} d\right)
$$

where the convolution $*$ follows from the independence between $N_{2}$ and $\beta X+(\beta / \alpha) D$.

\section{ApProximation to the PDF Models in the Presence OF SUBTRACTIVE DITHER}

Since (24) is very complex to implement, we make approximations to it. We can see that there are only two random variables involved in (24): $\widetilde{X}$ and $D$. Assuming that $\sigma_{\widetilde{X}}^{2} \gg \sigma_{D}^{2}$, we can approximate $f_{X+(1 / \alpha) D}(x)$ in the following way:

$$
\begin{aligned}
f_{X+\frac{1}{\alpha} D}(x) & =\sum_{k=-\infty}^{+\infty} f_{(1-\alpha) \widetilde{X}+k \Delta+\frac{1-\alpha}{\alpha} D}(x) I_{A_{k \mid W=0}}(x) \\
& \approx \sum_{k=-\infty}^{+\infty} f_{(1-\alpha) \widetilde{X}+k \Delta}(x) I_{A_{k \mid W=0}}(x) .
\end{aligned}
$$

Note that the output of the quantizer depends both on $\widetilde{X}$ and $D$, but since the variance of the first is assumed to be much larger, the term $k \Delta$ is present in the approximation together with $\widetilde{X}$. An illustration of $f_{X+(1 / \alpha) D}(x)$, its approximation as given by (26), and $f_{X}(x)$ is shown in Fig. 9. The difference between $f_{X+(1 / \alpha) D}(x)$ and its approximation can hardly be recognized. We can also see the huge difference between $f_{X+(1 / \alpha) D}(x)$ and $f_{X}(x)$.

\section{Design of the Dither Sequence}

In the previous section, we saw that $\sigma_{\widetilde{X}}^{2} \gg \sigma_{D}^{2}$ in order for (26) to be an accurate approximation. Approximation is perfect if $\sigma_{D}^{2}=0$, but this is unacceptable from security point of view. In this section, we find sufficient conditions for the dither sequence statistics such that, for $\sigma_{D}^{2}$ as small as possible, an attacker is not able to decode the watermark with an error probability $^{2}$ different than 0.5 .

\footnotetext{
${ }^{2}$ Since we have one-dimensional one-bit watermarking, the error probability and bit error probability are equal.
}

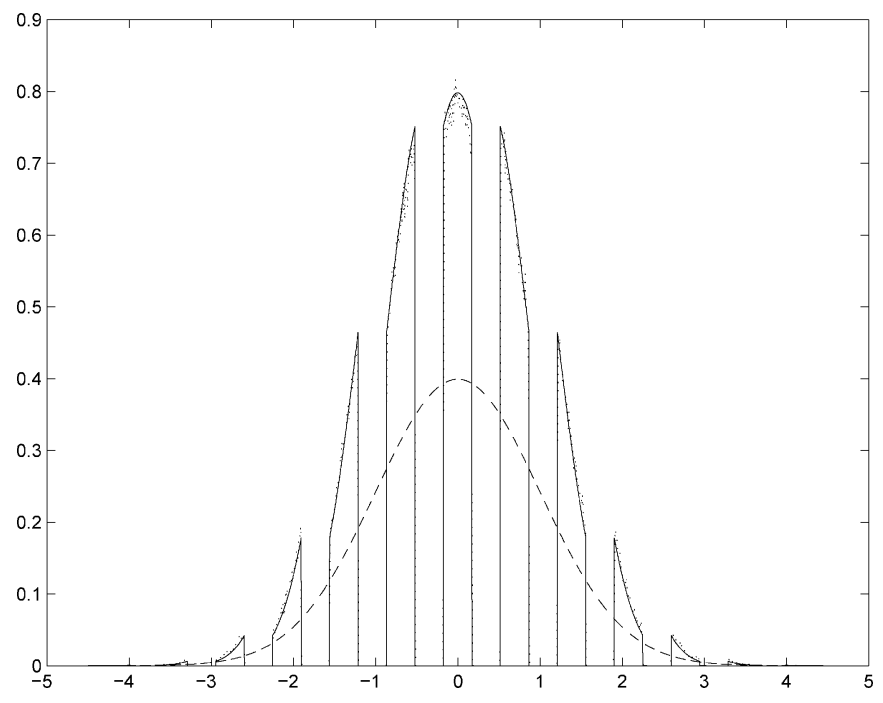

Fig. 9. An illustration of $f_{X}(x)$ (dashed line), $f_{X+(1 / \alpha) D}(x)$ (dotted line), and its approximation $\sum_{k=-\infty}^{+\infty} f_{(1-\alpha) \widetilde{X}+k \Delta}(x) I_{A_{k \mid W=0}}(x)$ (solid line). Chosen settings are $\tilde{X} \sim \mathcal{N}(0,1), \sigma_{N_{1}}^{2}=\sigma_{N_{2}}^{2}=\sigma_{D}^{2}=0.01$, and $\beta=1$.

To derive the conditions, we first need to derive the error probability, which is given by the following theorem.

Theorem 1: When the dither sequence $D$ is not known to the decoder, the error probability $P_{e}$ is given by the expression

$$
P_{e}=\sum_{m} \operatorname{Pr}\left[m \Delta-\frac{3 \Delta}{4} \leq(1-\alpha) N_{1}+\frac{\alpha}{\beta} N_{2}-D \leq m \Delta-\frac{\Delta}{4}\right]
$$

where $m \in(-\infty, \infty)$ is an integer.

Proof: The error probability $P_{e}$ can be expressed as

$$
\begin{aligned}
P_{e}= & \operatorname{Pr}[\hat{W}=1 \mid W=0] \operatorname{Pr}[W=0] \\
& +\operatorname{Pr}[\hat{W}=0 \mid W=1] \operatorname{Pr}[W=1] \\
= & \operatorname{Pr}[\hat{W}=1 \mid W=0]
\end{aligned}
$$

where the last line follows from the fact that the encoder is a symmetric scheme of two quantizers, that the channel strategy is independent of the embedded message, i.e., $f_{N_{2} \mid W}\left(n_{2} \mid w\right)=$ $f_{N_{2}}\left(n_{2}\right)$, and that $\operatorname{Pr}[W=0]+\operatorname{Pr}[W=1]=1$. Therefore, we can model the whole watermarking system, together with the attack channel, as a binary symmetric channel with crossover probability $P_{e}$.

From (28) and (6), it is straightforward to show that the probability of error when $D$ is not added at the decoder can be written as

$$
\begin{aligned}
P_{e} & =\operatorname{Pr}[\hat{W}=1 \mid W=0] \\
& =\operatorname{Pr}\left[|Q(\hat{U}-D)-(\hat{U}-D)| \geq \frac{\Delta}{4}\right] .
\end{aligned}
$$

Observe that for any $X$ and scalar quantizer $Q(\cdot)$ with step size $\Delta$, we can write the relation

$$
|Q(X)-X|=\left|\left(X+\frac{\Delta}{2}\right) \bmod \Delta-\frac{\Delta}{2}\right|
$$

Using (30) in (29), we have

$$
P_{e}=\operatorname{Pr}\left[\left|\left(\hat{U}-D+\frac{\Delta}{2}\right) \bmod \Delta-\frac{\Delta}{2}\right| \geq \frac{\Delta}{4}\right]
$$




$$
\begin{aligned}
=\operatorname{Pr} & {\left[\left(\hat{U}-D+\frac{\Delta}{2}\right) \bmod \Delta \leq \frac{\Delta}{4}\right.} \\
& \left.\bigcup\left(\hat{U}-D+\frac{\Delta}{2}\right) \bmod \Delta \geq \frac{3 \Delta}{4}\right]
\end{aligned}
$$

where $\bigcup$ denotes the union of two events.

Using (5) and taking into account that $U \in \Lambda$, the quantizer lattice, we can write the equation shown at the bottom of the page. Using number theory [17], we can write that for any $b$ and any $c$ such that $b>c>0$, and any $a \neq m b$, where $m \in$ $(-\infty,+\infty)$ is an integer, the solution to the inequalities

$$
\begin{aligned}
& a \bmod b \geq c \\
& a \bmod b \leq c
\end{aligned}
$$

is

$$
m b+c \leq a \leq(m+1) b
$$

and

$$
m b \leq a \leq m b+c
$$

respectively. Therefore, after simple arithmetic, we arrive at (27).

We would like to choose the dither sequence statistics such that the error probability $P e=0.5$ for all choices of the attacker noise $N_{2}$. We state the following theorem.

Theorem 2: For the probability of error $P_{e}=0.5$, it is sufficient to choose the dither uniformly distributed over the base quantization cell, ${ }^{3}$ i.e., $D \sim \mathcal{U}\left(0, \sigma_{N_{1}}^{2}\right)$.

Proof: For notational simplicity, we make the following substitution:

$$
Z=(1-\alpha) N_{1}+\frac{\alpha}{\beta} N_{2}-D
$$

We can express (27) in the following way:

$$
P_{e}=\sum_{m} \int_{m \Delta-3 \Delta / 4}^{m \Delta-\Delta / 4} f_{Z}(z) d z .
$$

By definition, we can write

$$
f_{Z}(z)=\iint f_{Z \mid N_{1}, N_{2}}\left(z \mid n_{1}, n_{2}\right) f_{N_{1}, N_{2}}\left(n_{1}, n_{2}\right) d n_{1} d n_{2}
$$

${ }^{3}$ In [14], it was shown that this is sufficient for making the quantization noise independent of the input signal.
Substituting with (39) in (38), we get

$$
\begin{aligned}
P_{e}= & \sum_{m} \int_{m \Delta-3 \Delta / 4}^{m \Delta-\Delta / 4} \iint f_{Z \mid N_{1}, N_{2}}\left(z \mid n_{1}, n_{2}\right) f_{N_{1}, N_{2}}\left(n_{1}, n_{2}\right) \\
& \times d n_{1} d n_{2} d z \\
= & \iint \sum_{m} \int_{m \Delta-3 \Delta / 4}^{m \Delta-\Delta / 4} f_{Z \mid N_{1}, N_{2}}\left(z \mid n_{1}, n_{2}\right) d z \\
& \times f_{N_{1}, N_{2}}\left(n_{1}, n_{2}\right) d n_{1} d n_{2}
\end{aligned}
$$

where in the second equality we interchanged the order of integration and summation.

From (37), we can write

$$
f_{Z \mid N_{1}, N_{2}}\left(z \mid n_{1}, n_{2}\right)=f_{D}\left((1-\alpha) n_{1}+\frac{\alpha}{\beta} n_{2}-z\right) .
$$

Therefore, $P_{e}$ can be written as

$$
\begin{aligned}
P_{e}=\iint \sum_{m} \int_{m \Delta-3 \Delta / 4}^{m \Delta-\Delta / 4} f_{D} & \left((1-\alpha) n_{1}+\frac{\alpha}{\beta} n_{2}-z\right) d z \\
& \times f_{N_{1}, N_{2}}\left(n_{1}, n_{2}\right) d n_{1} d n_{2} .
\end{aligned}
$$

From (41), we see that the term $(1-\alpha) n_{1}+(\alpha / \beta) n_{2}$ affects only the mean of $f_{D}(-z)$. If we choose the dither to be uniform over the base quantization cell, i.e., $D \sim \mathcal{U}\left(0, \sigma_{N_{1}}^{2}\right)$, then we can show that (see Fig. 10)

$$
\sum_{m} \int_{m \Delta-\frac{3 \Delta}{4}}^{m \Delta-\frac{\Delta}{4}} f_{D}\left((1-\alpha) n_{1}+\frac{\alpha}{\beta} n_{2}-z\right) d z=0.5 .
$$

Therefore

$$
P_{e}=\iint 0.5 f_{N_{1}, N_{2}}\left(n_{1}, n_{2}\right) d n_{1} d n_{2}=0.5 .
$$

Note that in the proof of the theorems, we do not need the assumption $\sigma_{\widetilde{X}}^{2} \gg \sigma_{D}^{2}$, and therefore the high resolution quantization assumption [10] is not necessary for the system security. However, we will assume the low distortion case $\sigma_{\widetilde{X}}^{2} \gg \sigma_{N_{1}}^{2}=$ $\sigma_{D}^{2}$ because of the approximation assumptions in the previous section.

Using Fig. 10, it can also be shown that $P_{e} \leq 2 / 3$, with equality when $\sigma_{D}^{2}=(9 / 4) \sigma_{N_{1}}^{2}$ and $\sigma_{N_{2}}^{2}=0$.

Experimental curves for the probability of error $P_{e}$ as a function of $\sigma_{D}^{2}$ for different values of $\sigma_{N_{2}}^{2}$ are shown in Fig. 11. It can be seen that $P_{e}=0.5$ independently of $\sigma_{N_{2}}^{2}$, as long as $D \sim \mathcal{U}\left(0, \sigma_{N_{1}}^{2}\right)$.

$$
P_{e}=\operatorname{Pr}\left[\left((1-\alpha) N_{1}+\frac{\alpha}{\beta} N_{2}-D+\frac{\Delta}{2}\right) \bmod \Delta \leq \frac{\Delta}{4} \bigcup\left((1-\alpha) N_{1}+\frac{\alpha}{\beta} N_{2}-D+\frac{\Delta}{2}\right) \bmod \Delta \geq \frac{3 \Delta}{4}\right]
$$




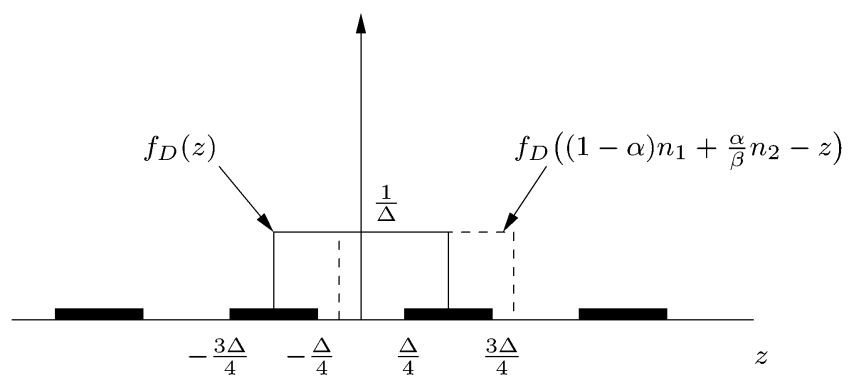

Fig. 10. An illustration of $\sum_{m} \int_{m \Delta-3 \Delta / 4}^{m \Delta-\Delta / 4} f_{Z \mid N_{1}, N_{2}}\left(z \mid n_{1}, n_{2}\right) d z$ for $D \sim$ $\mathcal{U}\left(0, \sigma_{N_{1}}^{2}\right)$.

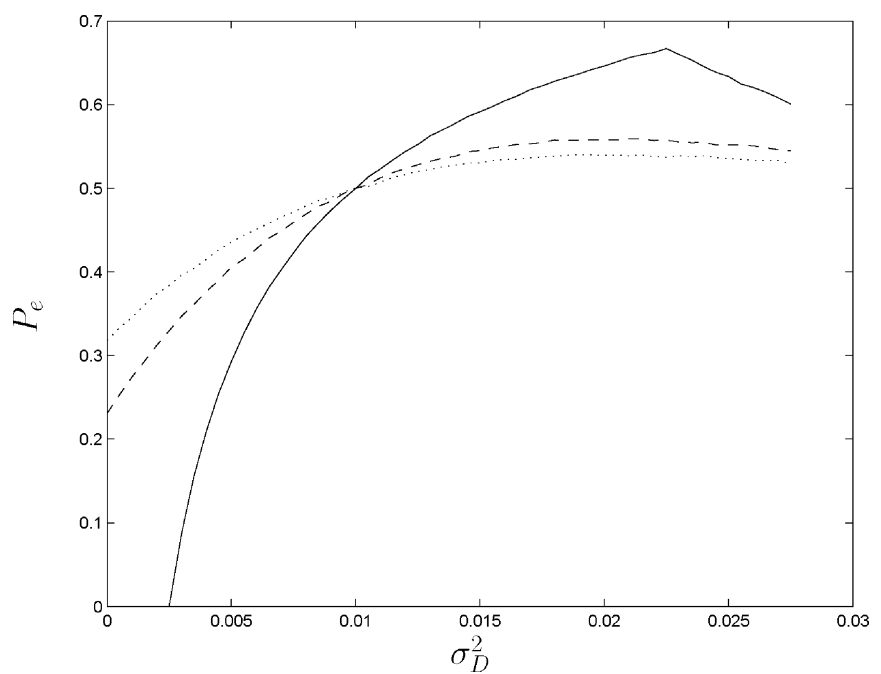

Fig. 11. Experimental curves for $P_{e}$ as a function of $\sigma_{D}^{2}$ for different values of $\sigma_{N_{2}}^{2}$. The solid curve is for $\sigma_{N_{2}}^{2}=0$, the dashed curve is for $\sigma_{N_{2}}^{2}=0.01$, and the dotted curve is for $\sigma_{N_{2}}^{2}=0.02$. Chosen settings are $\widetilde{X} \sim \mathcal{N}(0,1)$, $D \sim \mathcal{U}\left(0, \sigma_{D}^{2}\right), N_{2} \sim \mathcal{N}\left(0, \sigma_{N_{2}}^{2}\right), \sigma_{N_{1}}^{2}=0.01$, and $\beta=1$.

\section{ML Estimation of $\beta$}

The pdf models of the watermarked and attacked data have been derived as a function of $\beta$ in the previous sections. We are now able to use these models to estimate $\beta$ from the observed signal $Y$.

We assume that the host signal and attack channel noise are independent identically distributed (i.i.d.) vector sources, i.e., we consider all signals to be $n$-dimensional vectors with i.i.d. components. The ML estimation of $\beta$ is done based on the following relation:

$$
f_{Y+\frac{\beta}{\alpha} D}\left(y+\frac{\beta}{\alpha} d\right)=f_{\beta X+\frac{\beta}{\alpha} D}\left(\beta x+\frac{\beta}{\alpha} d\right) * f_{N_{2}}\left(n_{2}\right) .
$$

Representing $f_{Y+(\beta / \alpha) D}(y+(\beta / \alpha) d)$ as a joint distribution, the ML estimation $\hat{\beta}$ of the parameter $\beta[18]$ is given as

$$
\begin{aligned}
\hat{\beta} & =\arg \max _{\beta} f_{Y_{1}+\frac{\beta}{\alpha} D_{1}, \ldots, Y_{n}+\frac{\beta}{\alpha} D_{n}}\left(y_{1}+\frac{\beta}{\alpha} d_{1}, \ldots, y_{n}+\frac{\beta}{\alpha} d_{n}\right) \\
& =\arg \max _{\beta} f_{Y_{1}+\frac{\beta}{\alpha} D_{1}}\left(y_{1}+\frac{\beta}{\alpha} d_{1}\right) \ldots f_{Y_{n}+\frac{\beta}{\alpha} D_{n}}\left(y_{n}+\frac{\beta}{\alpha} d_{n}\right) \\
& =\arg \max _{\beta} \sum_{i} \log f_{Y_{i}+\frac{\beta}{\alpha} D_{i}}\left(y_{i}+\frac{\beta}{\alpha} d_{i}\right) .
\end{aligned}
$$

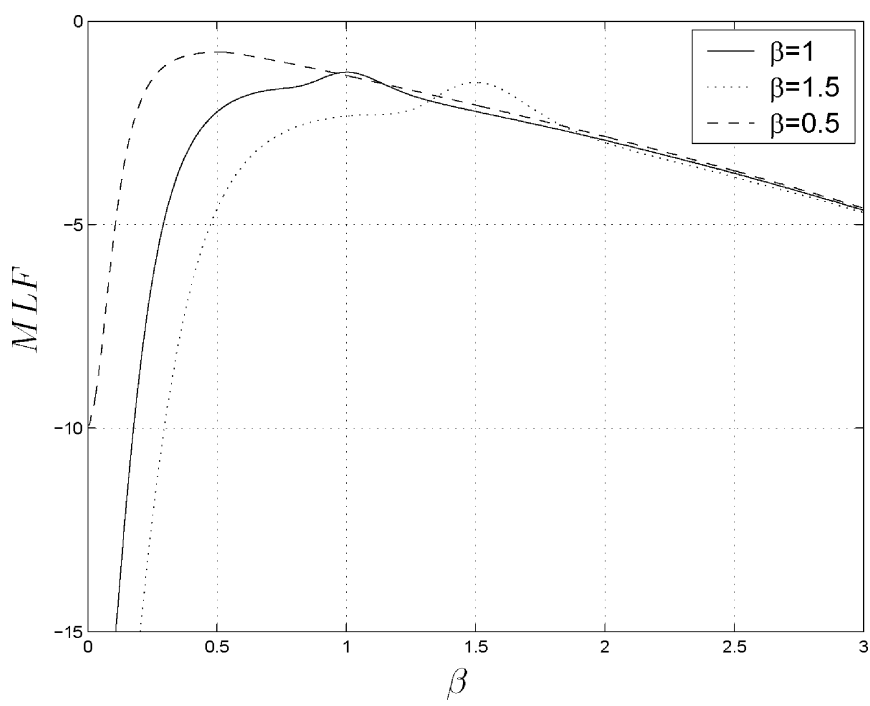

Fig. 12. Graph of MLF for different values of $\beta$. Chosen settings are $\widetilde{X} \sim$ $\mathcal{N}(0,1), D \sim \mathcal{U}(0,0.01), N_{2} \sim \mathcal{N}(0,0.01)$, and $\sigma_{N_{1}}^{2}=0.01$.

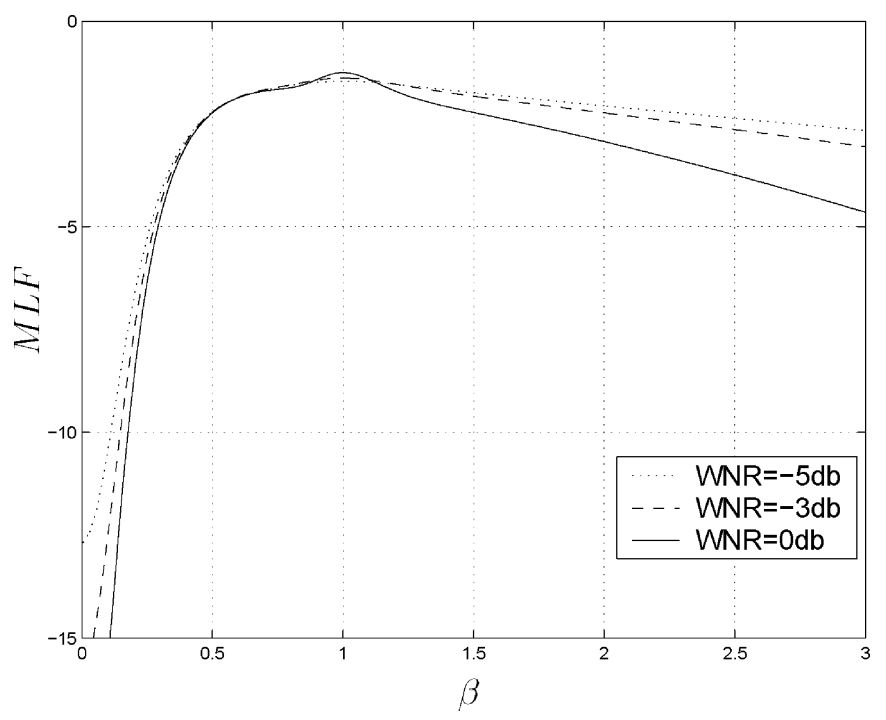

Fig. 13. Graph of MLF for different values of WNR. Chosen settings are $\widetilde{X} \sim$ $\mathcal{N}(0,1), D \sim \mathcal{U}(0,0.01), \sigma_{N_{1}}^{2}=0.01$, and $\beta=1$.

Here the second line follows from the assumption that the received data consist of $n$ i.i.d. samples, and therefore the joint pdf can be written as a product of the " $n$ " marginal pdfs. The last line follows from the monotonicity of the logarithm.

Experimental curves for the maximum likelihood functional (MLF), which is the expression $\sum_{i} \log f_{Y_{i}+(\beta / \alpha) D_{i}}\left(y_{i}+\right.$ $\left.(\beta / \alpha) d_{i}\right)$, are shown in Figs. 12 and 13.

\section{EXPERIMENTS}

In this section, we describe experiments carried out to test the estimation accuracy of the proposed technique in terms of WNR and the number of available signal samples $n$. In principle, one aims at developing estimation techniques that require a small amount of data, so that they can be applied in situations where the estimating parameter slowly varies. Since it is difficult to further manipulate (25) (even for Gaussian sources) because of 


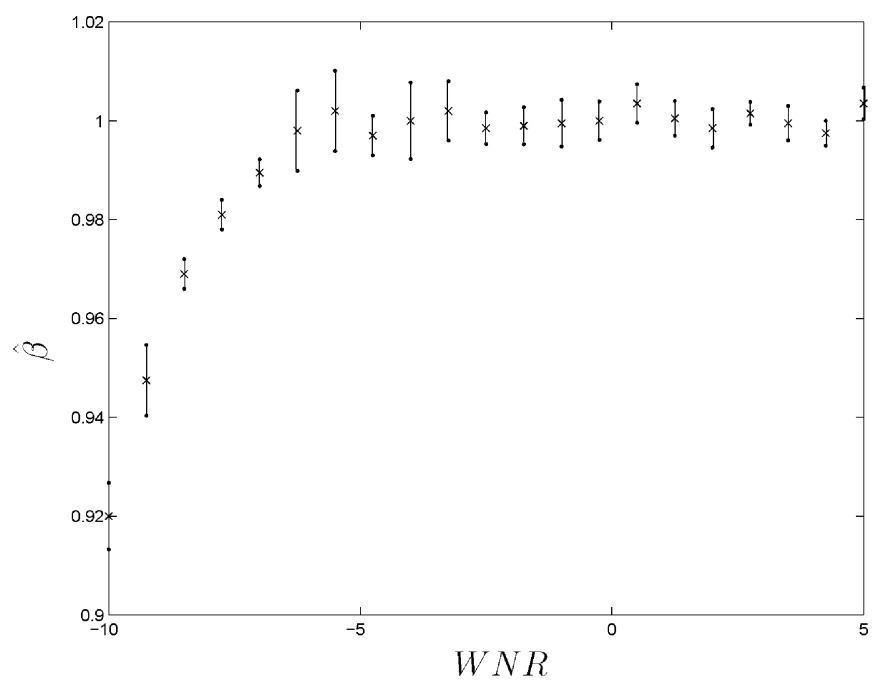

Fig. 14. Graphs of $\hat{\beta}$ as a function of WNR. The crosses represent the mean and the lines the standard deviation in both directions. The chosen settings are $\widetilde{X} \sim \mathcal{N}(0,1)$, DWR $=20 \mathrm{~dB}, \beta=1$, and $n=350000$.

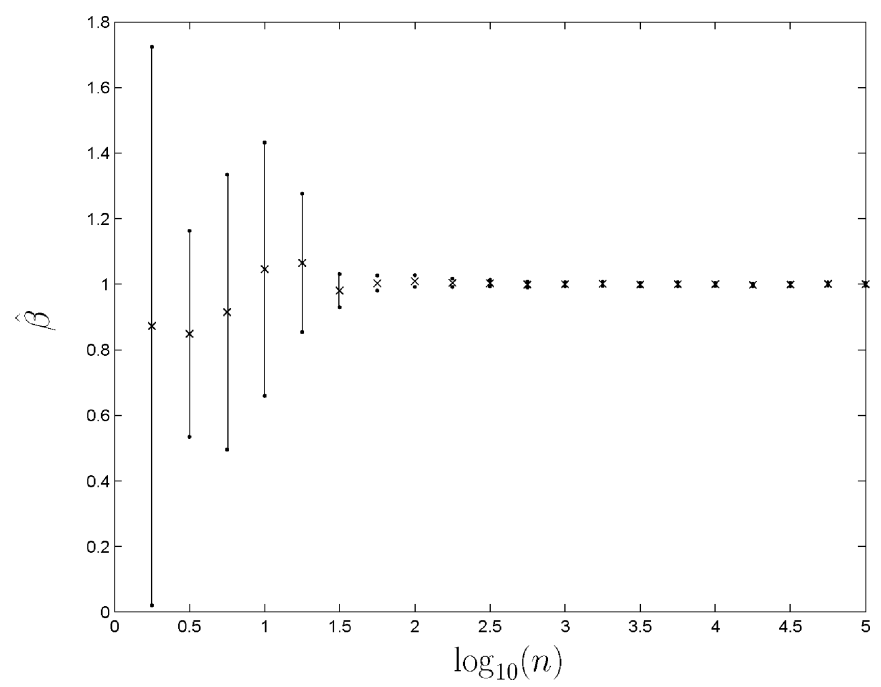

Fig. 15. Graphs of $\hat{\beta}$ for synthetic host signals as a function of the number of signal samples $n$. The crosses represent the mean and the lines the standard deviation in both directions. Chosen settings are $\widetilde{X} \sim \mathcal{N}(0,1)$, DWR $=20 \mathrm{~dB}$, $\mathrm{WNR}=0 \mathrm{~dB}$, and $\beta=1$.

the indicator function in $f_{\beta X+(\beta / \alpha) D}(\beta x+(\beta / \alpha) d)$, we do brute force search for the optimal $\beta$.

\section{A. Synthetic Host Signals}

Here we perform experiments with synthetic host signals. We assume that the estimator has perfect knowledge of the host signal variance. In Fig. 14, we present results for $\hat{\beta}$ as a function of WNR. It can be seen that for WNR $>-7 \mathrm{~dB}$, the mean of $\hat{\beta}$ is very close to the true value of $\beta$, and the standard deviation of $\hat{\beta}$ is always smaller than $1 \%$. In Fig. 15, we present results for $\hat{\beta}$ as a function of number of signal samples. It can be seen that around 100 signal samples are needed for reliable estimation of $\beta$. Results of $\beta-\hat{\beta}$ as a function of $\beta$ are presented in Fig. 16. We can see that the standard deviation of $\beta-\hat{\beta}$ is smaller than $1 \%$ for $\beta>0.75$.

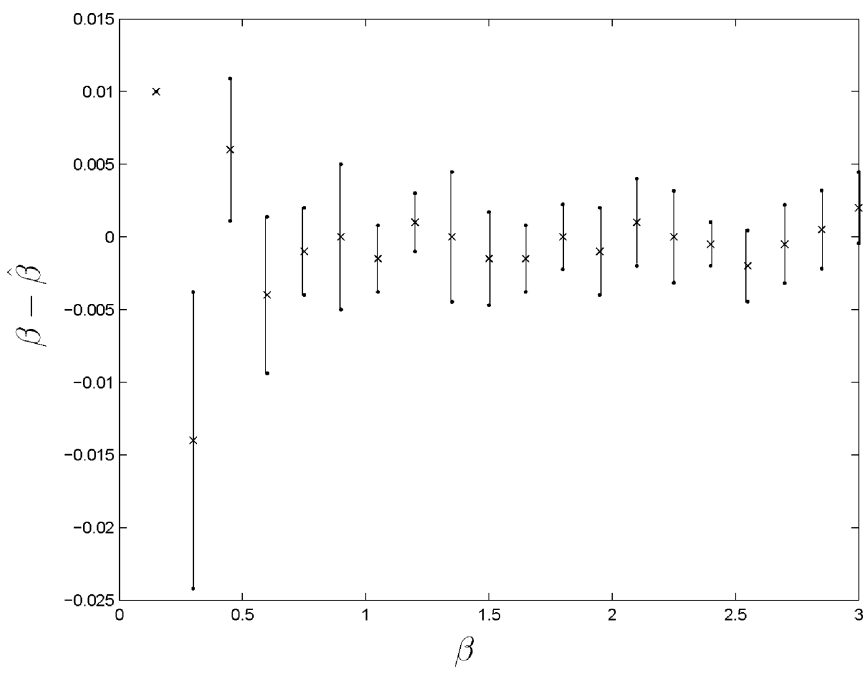

Fig. 16. Graphs of $\beta-\hat{\beta}$ as a function of $\beta$. The crosses represent the mean and the lines the standard deviation in both directions. Chosen settings are $\widetilde{X} \sim$ $\mathcal{N}(0,1), \mathrm{DWR}=20 \mathrm{~dB}, \mathrm{WNR}=0 \mathrm{~dB}, n=350000$.

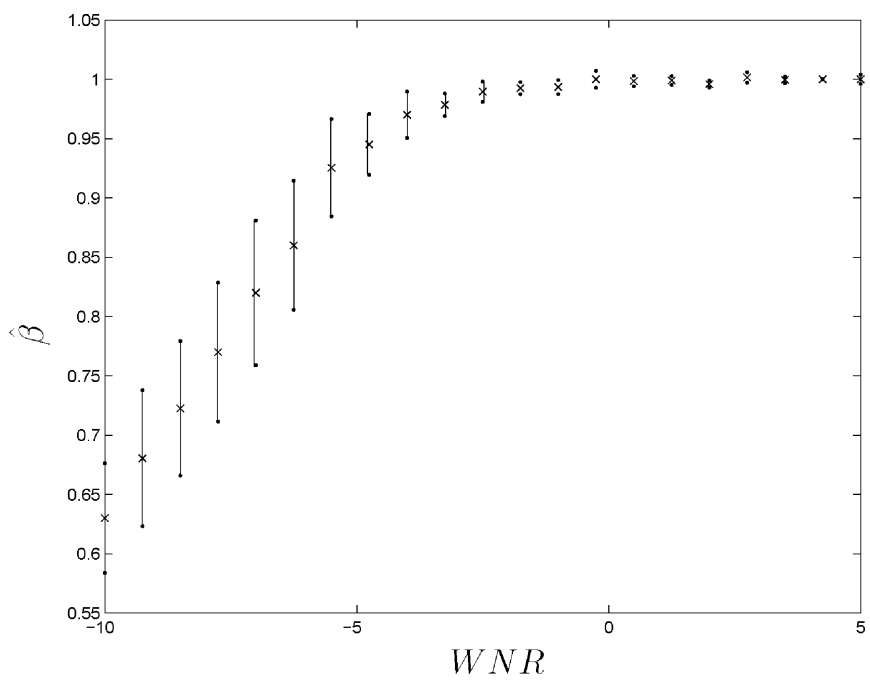

Fig. 17. Graphs of $\hat{\beta}$ for real audio signals as a function of WNR. The crosses represent the estimation mean and the lines the standard deviation in both directions. Chosen settings are DWR $=20 \mathrm{~dB}, \beta=1$, and $n=350000 \ldots 500000$.

\section{B. Real Host Signals}

In this subsection, we describe experiments with real audio signals (audio and speech with sampling frequency $48 \mathrm{kHz}$ ). We choose more realistic settings than in the case of synthetic hosts, in which the estimator does not have a perfect knowledge of the host signal variance. The assumed pdf model of the host signal at the detection side is a zero-mean Laplacian pdf with variance equal to the variance of the received signal, i.e., $\widetilde{X} \sim \mathcal{L}\left(0, \beta^{2}\left(\sigma_{\widetilde{X}}^{2}+\sigma_{N_{1}}^{2}\right)+\sigma_{N_{2}}^{2}\right)$. This is a realistic assumption because the decoder has access to the received data and can estimate its variance. Furthermore, in practice, most audio signals have a marginal pdf that resembles the Laplacian pdf [19]. Experimental results in terms of WNR are shown in Fig. 17. It can be seen that the standard deviation of $\hat{\beta}$ is smaller than $1 \%$ for $W N R>-5 \mathrm{db}$. Experimental results of $\hat{\beta}$ as a function of the number of signal samples are shown in Fig. 18. It can be seen 


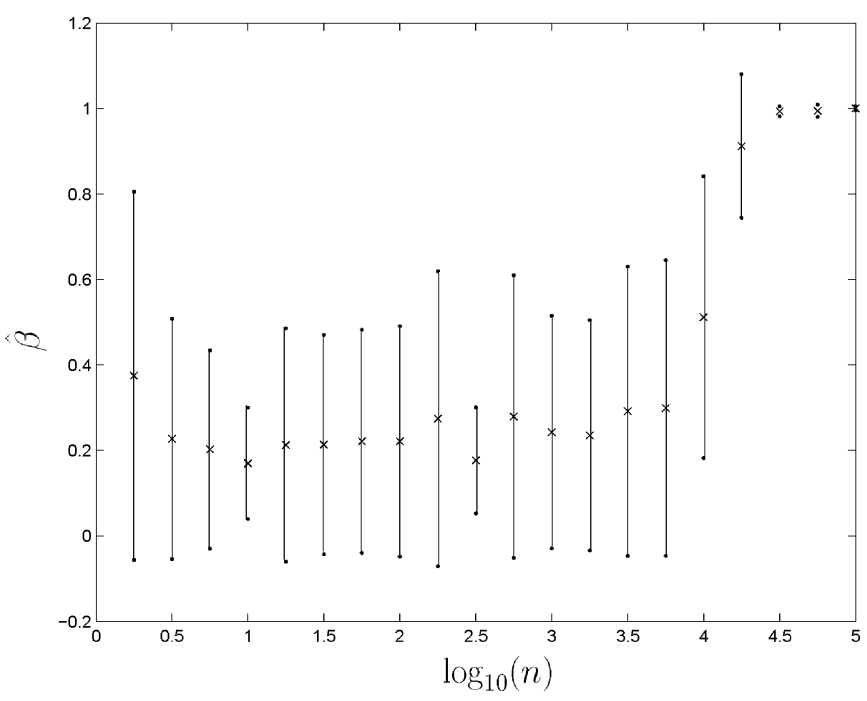

Fig. 18. Graphs of $\hat{\beta}$ for real audio signals as a function of the number of signal samples $n$. The crosses represent the mean and the lines the standard deviation in both directions. Chosen settings are DWR $=20 \mathrm{~dB}$, WNR $=0 \mathrm{~dB}$, and $\beta=1$.

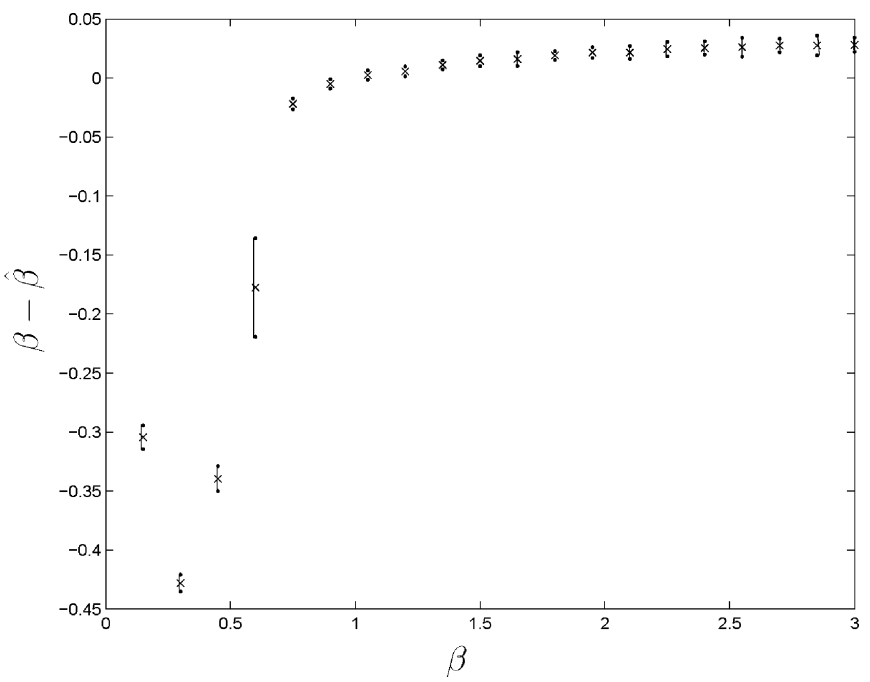

Fig. 19. Graphs of $\beta-\hat{\beta}$ for real audio signals as a function of $\beta$. The crosses represent the mean and the lines the standard deviation in both directions. Chosen settings are DWR $=20 \mathrm{~dB}$, WNR $=0 \mathrm{~dB}$, and $n=350000 \ldots 500000$.

that reliable estimation of $\beta$ is possible for $n>31000$ samples. In Fig. 19, we plot experimental results of $\beta-\hat{\beta}$ as a function of $\beta$ for different audio signals. It can be seen that the standard deviation of $\beta-\hat{\beta}$ is smaller than $1 \%$ for $\beta>0.75$.

The experimental results with real signals are generally worse than in the case of synthetic signals. There are several reasons for that. First, the experimental settings are different. For real signals the estimator has access only to the received signal. The variance of the received signal differs from the variance of the host signal, and the difference is especially pronounced when $\beta$ deviates from one. This causes a difference between the pdf of the host signal and the pdf assumed by the estimator. Second, real signals are nonstationary and exhibit correlation between the samples, which is not captured by our pdf models.
The ML estimation procedure is computationally very expensive, because of the brute force searching for the optimal $\beta$. Reference [20] treats the problem of jointly estimating $\beta$ and $\sigma_{N_{2}}^{2}$ by transforming the attack channel into one that is equivalent but computationally less expensive for the ML approach processing chain. However, this transform does not improve the estimation.

\section{CONCLUSION AND DISCUSSION}

We presented an ML amplitude scale estimation technique for quantization-based watermarking. We also incorporated subtractive dither into the watermarking system and gave sufficient conditions for the dither sequence to achieve a given level of security. The estimation approach needs a small amount of signal samples for estimating reliably $\beta$ in the case of synthetic host signals but a relatively large amount of signal samples in the case of real audio host signals. Experiments showed that the proposed approach performs well under realistic conditions.

\section{ACKNOWLEDGMENT}

The authors wish to acknowledge the helpful suggestions of the reviewers.

\section{REFERENCES}

[1] P. Moulin and J. A. O'Sullivan, "Information-theoretic analysis of information hiding," IEEE Trans. Inf. Theory, vol. 49, no. 3, pp. 563-593, Mar. 2003.

[2] B. Chen and G. Wornell, "Quantization index modulation: A class of provably good methods for digital watermarking and information embedding," IEEE Trans. Inf. Theory, vol. 47, pp. 1423-1443, May 2001.

[3] S. I. Gel'fand and M. S. Pinsker, "Coding for channel with random parameters," Prob. Contr. Inf. Theory, vol. 9, pp. 19-31, 1980.

[4] M. H. Costa, "Writing on dirty paper," IEEE Trans. Inf. Theory, vol. IT-29, no. 3, pp. 439-441, May 1983.

[5] J. H. Conway and N. J. A. Sloane, Sphere Packings, Lattices and Groups, 3rd ed. Berlin, Germany: Springer-Verlag, 1999.

[6] M. L. Miller, G. J. Doerr, and J. Cox, "Dirty-paper trellis codes for watermarking," in IEEE Int. Conf. Image Process., Rochester, NY, Sep. 2002, vol. 2, pp. 129-132.

[7] B. Bradley, "Improvement to CDF grounded lattice codes," in Proc. SPIE Security, Steganography, Watermarking Multimedia Contents VI, Jan. 2004, vol. 5306.

[8] F. Perez-Gonzalez, C. Mosquera, M. Barni, and A. Abrardo, "Rational dither modulation: A high rate data-hiding method invariant to gain attacks," IEEE Trans. Signal Process., vol. 53, no. 10, pp. 3960-3975, Oct. 2005.

[9] J. J. Eggers, R. Bauml, and B. Girod, "Estimation of amplitude modifications before SCS watermark detection," in Proc. SPIE Security Watermarking Multimedia Contents IV, San Jose, CA, Jan. 2002, vol. 4675, pp. 387-398.

[10] I. D. Shterev and R. L. Lagendijk, "Maximum likelihood amplitude scale estimation for quantization-based watermarking in the presence of dither," in Proc. SPIE Security, Steganography, Watermarking Multimedia Contents VII, Jan. 2005.

[11] I. D. Shterev, R. L. Lagendijk, and R. Heusdens, "Statistical amplitude scale estimation for quantization-based watermarking," in SPIE Security, Steganography, Watermarking Multimedia Contents VI, Jan. 2004, vol. 5306.

[12] K. Lee, D. S. Kim, T. Kim, and K. A. Moon, "EM estimation of scale factor for quantization-based audio watermarking," in Int. Workshop Digital Watermarking, Seoul, Korea, Oct. 2003.

[13] F. Balado, K. M. Whelan, G. C. M. Silvestre, and N. J. Hurley, "Joint iterative decoding and estimation for side-informed data hiding," IEEE Trans. Signal Process., vol. 53, no. 10, pp. 4006-4019, Oct. 2005.

[14] L. Schuchman, "Dither signals and their effect on quantization noise," IEEE Trans. Commun. Technol., vol. COM-12, no. 4, pp. 162-165, Dec. 1964.

[15] J. J. Eggers and B. Girod, Informed Watermarking. Norwell, MA: Kluwer Academic, 2002. 
[16] J. J. Eggers, J. K. Su, and B. Girod, "A blind watermarking scheme based on structured codebooks," in Inst. Elect. Eng. Colloq. Secure Images Image Authentication, London, U.K., Apr. 2000.

[17] K. Chandrasekharan, Introduction to Analytic Number Theory. Berlin, Germany: Springer-Verlag, 1968.

[18] H. V. Poor, An Introduction to Signal Detection and Estimation, 2nd ed. Berlin, Germany: Springer-Verlag, 1994.

[19] S. Gazor and W. Zwang, "Speech probability distribution," IEEE Signal Process. Lett., vol. 10, no. 7, pp. 204-207, Jul. 2003.

[20] R. L. Lagendijk and I. D. Shterev, "Estimation of attacker's noise and variance for QIM-DC watermark embedding," presented at the IEEE Int. Conf. Image Process., Singapore, Oct. 2004.

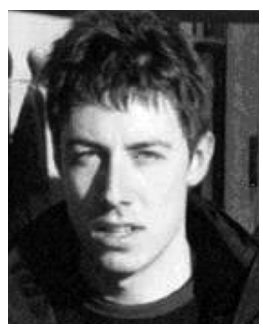

Ivo D. Shterev received the M.Sc. degree in electronics from the Technical University of Sofia, Plovdiv, Bulgaria, in 1999. He is currently pursuing the $\mathrm{Ph} . \mathrm{D}$. degree in the Information and Communication Theory Group, Delft University of Technology, Delft, The Netherlands.

He did a summer 2004 internship at the University of Illinois at Urbana-Champaign. His research interests include probability theory, information theory, and statistics.
Reginald L. Lagendijk (S'87-M'90-SM'97) received the M.Sc. and Ph.D. degrees in electrical engineering from Delft University of Technology, Delft, The Netherlands, in 1985 and 1990, respectively.

He became an Assistant Professor at Delft University of Technology in 1987. He was a Visiting Scientist in the Electronic Image Processing Laboratories, Eastman Kodak Research, Rochester, NY, in 1991 and Visiting Professor at Microsoft Research and Tsinghua University, Beijing, China, in 2000 and 2003 , respectively. Since 1999, he has been full Professor in the Information and Communication Theory Group, Delft University of Technology. He is author of Iterative Identification and Restoration of Images (Norwell, MA: Kluwer, 1991) and a coauthor of Motion Analysis and Image Sequence Processing (Norwell, MA: Kluwer, 1993) and Image and Video Databases: Restoration, Watermarking, and Retrieval (Amsterdam, The Netherlands: Elsevier, 2000). He was Associate Editor of Signal Processing: Image Communication. He has been involved in the conference organizing committees of ICIP2001, 2003, 2006, and 2011. At present his research interests include signal processing and communication theory, with emphasis on visual communications, compression, analysis, searching, and security. He is currently leading and actively involved in a number of projects in the field of intelligent information processing and data hiding for ad hoc and peer-to-peer multimedia communications.

Prof. Lagendijk was Associate Editor of the IEEE TRANSACTIONS ON Image Processing and IEEE TRANSACtions on Signal Processing's Supplement on Secure Digital Media. He is currently Associate Editor of the IEEE TRANSACTIONS ON INFORMATION FoRENSICS AND SECURITY. He was a member of the IEEE Signal Processing Society's Technical Committee on Image and Multidimensional Signal Processing. 